\title{
Études onomasiologiques et sémasiologiques des technolectes et des unités terminologiques complexes
}

\author{
Emmanuel Aito/Chidi Igwe (Regina, Canada)
}

\begin{abstract}
Terminology is strengthening its foundations as a sub-discipline through reflections on its principles and methodologies by entrenching the study of its principal object, to wit the term (Boulanger et L'Homme 1991). Thus, a constellation of theories and innovative methods have been suggested by contributors such as Temmerman (2000) who opts for a sociocognitive approach by applying the tenets of cognitive semantics to ontologies in the life sciences; Cabré $(1998,2000)$ who isolates the polyhedric nature of the term; Diki-Kidiri $(1999,2000)$ whose treatment of the cultural dimensions of the term is especially pertinent to less described languages; and Kageura (1995) whose semantic treatise delineates the boundaries of "conceptology". Collectively, these works apprehend the term contre-jour, in particular as a unit of comprehension in relation to its immediate environment, in contradistinction to its traditional definition which in the classical mould considers a term as contextually stripped of its textual or discursive value. In sequel of recent theoretical trends therefore, this paper represents our initial theoretical attempt to put into relief semasiological and onomasiological approaches to the term/concept association, in order to shed additional light on their possible complementarity with respect to both lexicographical and terminographical definitions of the term.
\end{abstract}

\section{$1 \quad$ Introduction}

Pour mieux asseoir ses principes et ses méthodes, le domaine de la terminologie connaît de nos jours d'importantes transformations dans ses pratiques et théories par rapport à ses débuts dans les années 30. La filiation de l'encadrement de ses outils épistémologiques et de sa description fait montre d'une richesse et de grandes variétés. Cependant, ce n'est qu'en 1985 (documenté en 1988: 27) que Pierre Auger emploie le mot «téméraire » pour s'autoriser le projet de dresser le bilan général des réalisations de la terminologie dans le monde et au Québec jusqu'alors, réservant à la fin de son allocution une vue prospective tournée, bien sûr, vers le futur. En aval de sa rétrospective traçant l'histoire de la terminologie, jusqu'à sa genèse, de sa place dans l'histoire des idées, il révèle une typologisation des grandes tendances contemporaines de l'époque, y compris le postulat selon chaque école. La perspicacité d'Auger (1988) demeure encore indéniable à bien des égards quant aux progrès dans le champ d'études terminologiques. Quant à nous, il s'agit de tenter par la suite une première théorisation de la complémentarité des deux approches onomasiologique et sémasiologique en terminologie. Cela nous procurera le soubassement pour le dépouillement du corpus éventuel qui visera l'exemplification de nos axiomatisations. Pour y parvenir, nous nous appuyons en partie sur la géométrie sémantique de Gordon (1994).

Loin de tenter ici une déontologie pour la terminologie, domaine qui connaît actuellement un tempo remarquable (Auger 1988 [1985], Cabré 1998, Rey 1988 [1985], Temmerman 2000), il appert qu'il y a encore lieu de rappeler les dimensions centrifuges et centripètes du rapport complexe entre la forme linguistique et son contenu. Depuis ces dernières années que la ter- 
minologie connaît son essor, voire son développement systématique au fur et à mesure qu'elle mène une réflexion profonde sur ses principes, ses fondements, et ses méthodes, elle parvient à une plus grande acceptation, engendrant par la suite une acception et une reconnaissance de sa pertinence à la fois linguistique, sociale et politique, tant sur le plan national qu'international (Cabré 1998). Par ailleurs, il y en a pour souhaiter un investissement de ses apports concrets, pourvu qu'elle passe du stade amateur au stade scientifique autonome, car « elle possède maintenant les indispensables fondements théoriques et méthodologiques qui personnalisent une science » (Boulanger et L'Homme 1991: 23).

Cette maturité qu'attribuent des partisans à la terminologie contemporaine lui procure la foi qui lui permet de sortir de ses confins autrefois casaniers, dans le but de se faire valoir en d'autres sciences du langage. À titre d'exemple, nous nous proposons dans nos présentes réflexions de faire allusion aux zones frontalières entre la sémasiologie, réservée jusqu'ici en terminologie à la lexicologie, et l'onomasiologie, approche qui convient à la terminologie traditionnelle wüsterienne. Sans vouloir lui dérober son utilité, la pratique onomasiologique constitue le point de départ où ancrer la complémentarité éventuelle des deux perspectives onomasiologiques et sémasiologiques du rapport terme/concept en terminologie. Est-ce vouloir avancer la conviction que la terminologie est maintenant « science à plein temps » comme le demande Kocourek (1994: 14) ? Pour répondre, certaines conditions s'imposent telles qu'elles sont présentées dans les réflexions historiques, épistémologiques et appréciatives : la terminologie, fait-elle preuve d'une prise de conscience de son propre univers ? Saisit-elle bien son aspect historique, épistémologique, pragmatique et éthique ? Exploite-t-elle avec succès ses liens avec d'autres sciences connexes ? Voilà un échantillon des questions auxquelles répondent les interrogations des intéressés.

La terminologie étant une activité dynamique, plutôt qu'un état inébranlable, il y a lieu de l'appréhender par sa nature multidimensionnelle. Les dimensions linguistiques et communicatives de la terminologie, faits renforcés par la polyvalence des termes qui y sont inhérents, conduisent aux caractères cognitifs et psychiques. Le domaine et ses multiples objets se développent grand'erre, changeant, et se déplaçant. Le stockage et la transmission des connaissances dans un discours spécialisé se déroulent concrètement dans de véritables situations de communication. C'est là justement où réside sa pratique sociale, au-delà des enclos des spécialistes. La terminologie, tout en véhiculant les concepts organisés textuellement dans un domaine donné au sein d'une société, ne saurait trop s'éloigner de celle-ci, ni de ses idéologies, enjeux et fonctions qui se prêtent à une légitime discussion (Rey 1985). Les termes, peu importe les domaines où ils apparaissent, demeurent des unités à trois dimensions, à savoir les dimensions formelle, conceptuelle et fonctionnelle. Ce dernier constat les situe nécessairement dans une négociation communicative, ce qui confère aux termes leur caractère pragmatique dans des situations concrètes de communication (Cabré 1998).

\section{Quelques perspectives novatrices}

Quatre tendances que nous jugeons représentatives des dernières axiomatisations méritent notre attention dans les paragraphes qui suivent. Tantôt convergentes, et tantôt divergentes, ces approches signalent, dans leur ensemble, des voies intéressantes.

\subsection{Sociocognitivisme (Temmerman)}

Profitant des deux tendances onomasiologique et sémasiologique, Temmerman (2000a: 58) privilégie la catégorisation dans le but de mieux élaborer une terminographie plus « réaliste des sciences de la vie ». C'est ce qui l'amène à établir une «théorie de la terminologie descriptive fondée sur des principes sociocognitifs ». Ses méthodes d'investigation s'inspirant de la sémantique cognitive, Temmerman (2000) puise dans un corpus extrait des publications 
scientifiques dans les sciences de la vie. Elle remet essentiellement en cause les fondements traditionnels de la théorie terminologique qui, selon elle, entrave une description plus réaliste et pragmatique d'un nombre important de catégories ontologiques et de termes. Ce qu'elle propose, et ce que nous trouvons d'une grande utilité pour les langues dans lesquelles les processus de conceptualisation restent encore à systématiser, réside dans l'adoption simultanée de l'onomasiologie et de la sémasiologie. Son approche ne nie pas non plus la présence inéluctable de la polysémie en langue spécialisée, ni l'apport de la diachronie qu'elle trouve incontournable pour aboutir aux finalités de son étude. En fait, la synchronie et la diachronie assistent, toutes deux, l'utilisateur de la terminologie à s'en servir à ses propres fins. Point n'est donc besoin de rationaliser notre prédilection envers la conception de Temmerman afin de nous attaquer aux défis de la dénomination et de la conceptualisation en ésan, langue minoritaire nigériane pour laquelle nous envisageons ultérieurement l'élaboration d'un lexique spécialisé.

Il est quelques distinctions qu'établit Temmerman (2000a: 59) entre la théorie traditionnelle viennoise et la théorie sociocognitive. Alors que la première part d'une notion bien délimitée, la seconde privilégie plutôt « les unités de compréhension» marquées le plus souvent par une structure archétypique. Deuxièmement, l'attribution de chaque notion a sa place dans le réseau conceptuel, alors que la théorie cognitive préconise la compréhension en tant que "événement structuré » qui se chevauche entre les catégories. Troisièmement, dans la théorie traditionnelle, la notion peut se retrouver à l'intérieur d'une «définition intensionnelle », alors que l'optique sociocognitive favorise l'unité de compréhension dont la définition variera. Ensuite, le terme et la notion qu'on lui attribue jouissent d'une permanence, entretenant donc une relation biunivoque, tandis que la théorie sociocognitive n'évacue pas la synonymie et la polysémie, d'où la nécessité de les décrire. Enfin, la synchronie et l'arbitraire, en dépit des cas de motivation, s'opposent traditionnellement à « l'évolution permanente », aux variations chronolectales et technolectales et à la métaphore (par exemple, celle anthroponymique). Bref, ils n'acceptent guère la présence de la motivation dans les modèles sociocognitifs.

\subsection{Théorie des portes (Cabré)}

Pour ce qui est de la théorie des portes (Cabré 2000), il s'agit de s'émanciper de l'interrogation coutumière au profit d'une orientation serrée, plus ciblée, préconisant le caractère polyédrique du terme. Cela enracinera sa pertinence dans plusieurs domaines sous-jacents, y compris la linguistique, chacun employant ses propres moyens. Cabré (2000: 10) qualifie cette ouverture de «système coopératif intégré de plusieurs théories [pour] décrire les unités terminologiques ». Citant Felber (1987: 87), Cabré (1998: 80) rapproche la terminologie des sciences cognitives en soulignant le fait que la cognition, résultat du processus psychique, a des retentissements importants sur la connaissance, processus qui relève du sujet de façon individuelle. Ce qui fait que l'individu se servant du terme porteur d'une notion ou de plusieurs notions peut non seulement s'exprimer et véhiculer des pensées dans un domaine, mais il pourra aussi structurer celui-ci à sa façon, tout en restant aux confins de l'ordre déterminé par les catégories ontologiques, sans que le domaine ne soit chaotique ni déstructuré. Pour sa part, Cabré (2000) trouve coïncidence entre communication spécialisée et communication générale, et par conséquent, elle ne voit guère d'opposition entre connaissance spécialisée et connaissance générale. Comme corollaire logique, elle estime que la terminologie ne peut pas «être expliquée de manière autonome, indépendamment des autres signes de la langue naturelle », en même temps que la terminologie aura du mal à se libérer des théories visant « à expliquer la communication et la cognition », d'où la nécessité de décrire le caractère interdisciplinaire du terme.

Les unités terminologiques, ajoute Cabré (2000: 15), peuvent être décrites selon « la grammaire d'une langue (phonologie, morphologie, lexique, syntaxe des phrases et texte). En cela, 
elle regagne effectivement Auger et L'Homme (1994: 17-21) qui déconseillent l'isolement et l'exclusivité réciproques entre lexicographie et terminographie. Pour ce faire, Cabré recommande d'inclure les règles et conditions formelles, y compris certains éléments de la sémantique cognitive afin d'atteindre « le caractère polyédrique des termes ».

\subsection{Conceptologie : Le concept dans la définition du terme (Kageura)}

Des deux points de vue onomasiologique et sémasiologique, le concept est incontestablement central dans l'étude des termes. Il occupe une place importante dans la définition, l'étude et la caractérisation du terme parce que, du point de vue onomasiologique, le terme n'est qu'une représentation lexicale du concept. Sur le plan de la terminologie systématique, semble-t-il, une unité sémantique ne devient un terme que lorsque celui-ci désigne un concept. Le fait que l'étude terminologique des termes place le concept au rang privilégié, c'est-à-dire qu'elle le considère comme la voie inéluctable à la dénomination et à sa définition, ajoute foi à sa prédilection envers l'onomasiologie. Par conséquent, la terminologie adopte classiquement une approche partant du concept vers la forme qui le désigne.

La conceptologie (nous traduisons commodément conceptology de Kageura) comprise en tant que science qui étudie le concept est perçue comme une démarche importante de la terminologie par Felber (1984: 103, Kageura 1995: 26). Elle étudie l'aspect sémantique du terme, approche à la fois sémantique et lexicale puisqu'elle se préoccupe exclusivement du concept en le reliant à l'unité lexicale qui le dénomme. Felber (1984, cf. Kageura 1995) renforce ce fait en postulant que la conceptologie ressemble à la sémantique, compte tenu de sa place en linguistique. Certes, l'assignation professionnelle d'un terme à un concept se fait consciemment, car elle est précédée d'une série d'activités méthodologiques formelles. D'abord, il faut vérifier si tel terme est à même de fournir une représentation adéquate. Cela implique une évaluation conceptuelle de la forme lexicale à plusieurs niveaux pour s'assurer de sa convenance morphologique, phonologique, et conceptuelle. Du point de vue de l'implantation, ce fait, en soi, est révélateur de l'éventualité d'une terminologie se dotant d'une démarche à la fois onomasiologique et sémasiologique.

Quant à l'optique onomasiologique, on peut délimiter un concept en le séparant des autres concepts, et établir les caractères de ce même concept. Par ailleurs, il est difficile d'analyser les relations qu'entretient un terme avec d'autres termes selon une approche onomasiologique parce qu'elle se veut biunivoque, ce qui conduit à la difficulté inhérente à l'adaptabilité et à la maniabilité d'un terme dans le système linguistique général, ce dont jouit normalement un item lexical. Mais pour parvenir à la vraie image du caractère d'un terme par rapport aux autres termes du système terminologique, il faut aussi une approche terminologique ayant la forme du terme comme point de départ. Pour renforcer cette proposition, nous réexaminons, ne serait-ce que sommairement, les soixante ans de géométrie sémantique tracées par Gordon (1992, 1994), dont nous adapterons neuf schémas à la sémiotique du rapport entre l'unité lexical et son contenu. Les réflexions, commentaires, critiques, malentendus, modifications et illuminations sur le fameux triangle d'Ogden et Richards (1923) nous paraissent instructifs à cet égard. 


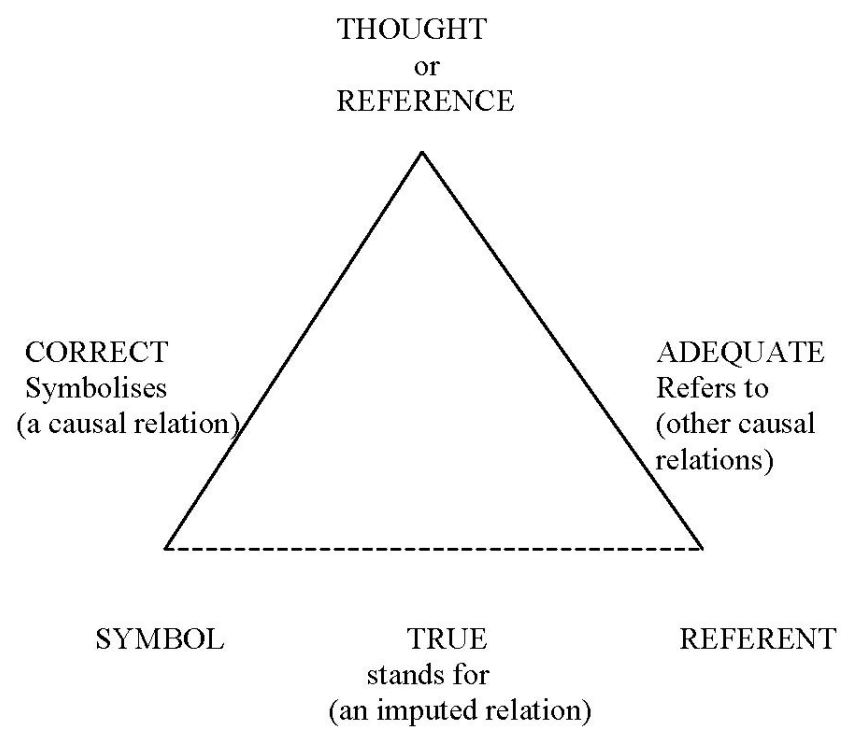

Figure 1

\subsubsection{Complexité inhérente de la dynamique forme et idée}

Selon Figure 1, le symbole et le référent se lient par une ligne pointillée, ce qui infère un manque de causalité, bien qu'elle y soit imputable, vu le rapport éventuel entre la symbolisation et la référentialité qui émane de la contextualisation. De fait, la causalité ne s'évacue guère de la théorie contextuelle de la signification. La prochaine figure, (Gordon 1994: 336) évoque simultanément l'existence parallèle et la distinction des deux contextes externe et psychologique. Selon Gordon, pour ce qui est de la base du triangle (Figure 2), c'est que « en effet, le facteur de causalité ne se laisse pas détacher de la théorie contextuelle de la signification ». Russo (1989: 12) abonde dans ce sens en avouant que c'est plutôt dans le contexte que le mot puisse retrouver sa signification. De la schématisation un peu fulgurante et flamboyante d'Oesterle (cf. Gordon 1992: 338) que nous n'avons pas reproduite, on passe à celle de Klaus Heger (Figure 3) que critique Schogt (1977: 35) en ce sens que «le désavantage de ce modèle est qu'il suggère un lien étroit entre le sème (ou le noème) et la classe de dénotés ». Pour remédier à la substance de ce reproche, Heger offre le prochain schéma (Figure 4), ce qui est censé éclairer simultanément les analyses paradigmatiques et syntagmatiques. Mais Gordon (1992: 340) trouve irrésistible la nécessité de signaler aux intéressés qu'il faut éviter « la fausse supposition d'un passage sur les axes du trapèze, qu'il existe un axe vertical implicite qui exclut la possibilité de multiplier les triangles à l'intérieur ». Selon lui, le trapèze revoie au symbole d'Ogden et Richards, et non pas à leur signe. La Figure 5 (de Hockett 1977) remplace le seul triangle de The Meaning of Meaning par quatre autres qui y sont implicitement associés; le triangle est dissimulé à l'intérieur du premier schéma (Figure 3) de Heger. Celui de Borella (1989: 156, la Figure 6) que qualifie Gordon (1992: 342) de «triangle à l'envers » postule que « les termes de la triangulation sémantique ne définissent le signe que s'ils sont envisagés dans les relations qui les unissent ». C'est ce qui nous amène à la conclusion que les "côtés du triangle comptent autant, sinon plus, que les sommets, qui n'existent que comme résultats de leur intersection ». Les modifications qu'apporte Borella (1989: 173) dans son second schéma tétradique (Figure 7) complètent le premier, triadique, pour distinguer les deux sens situés à chaque extrémité de l'axe vertical, allant alors à l'encontre de la conception saussurienne de base. 


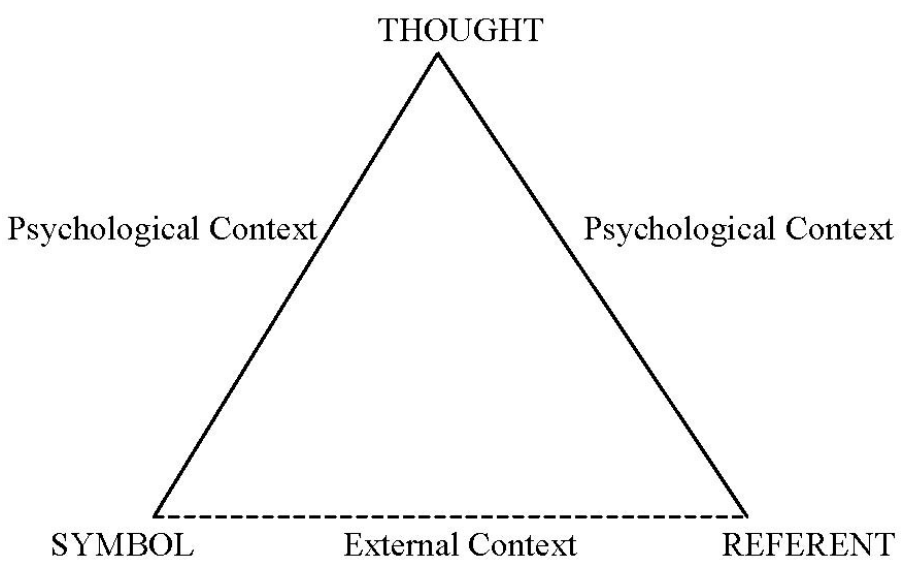

Figure 2

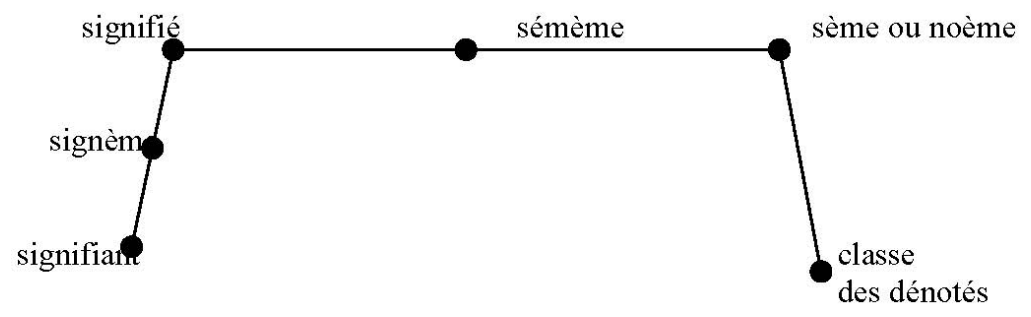

Figure 3

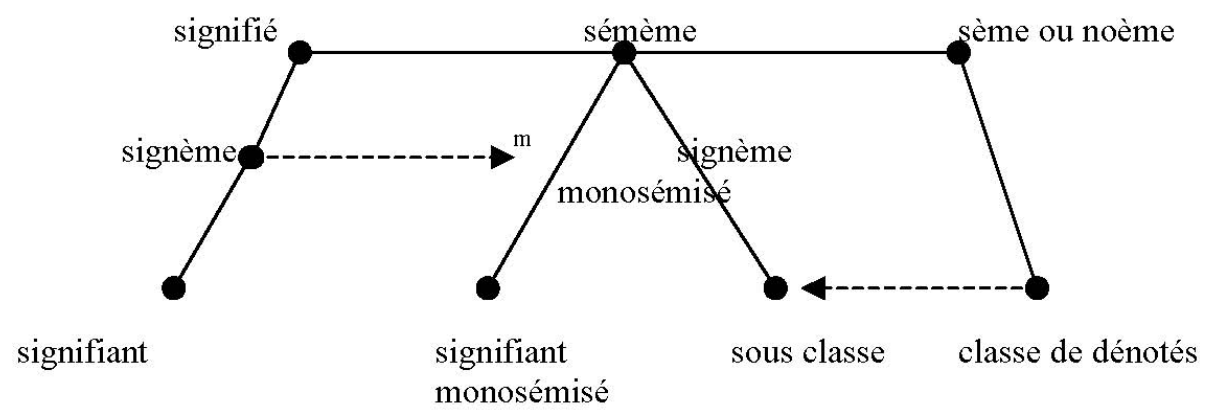

Figure 4

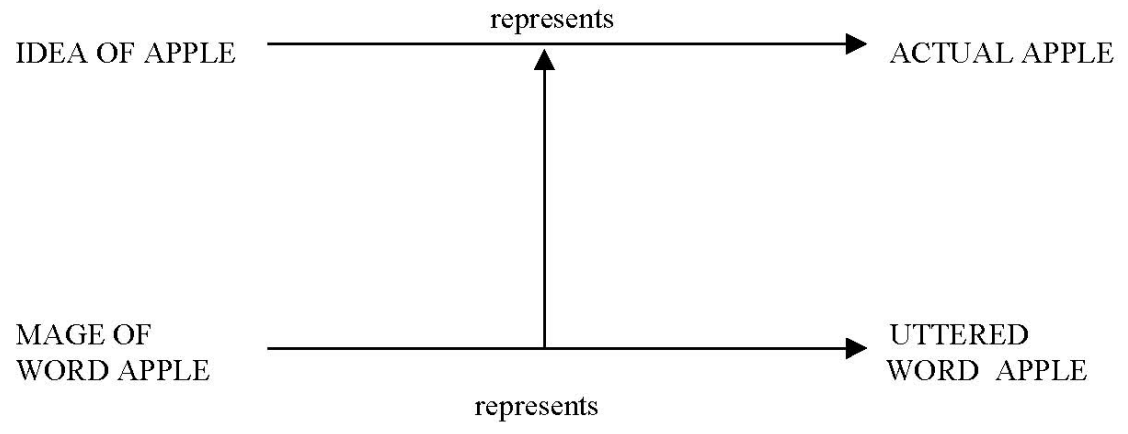

Figure 5 


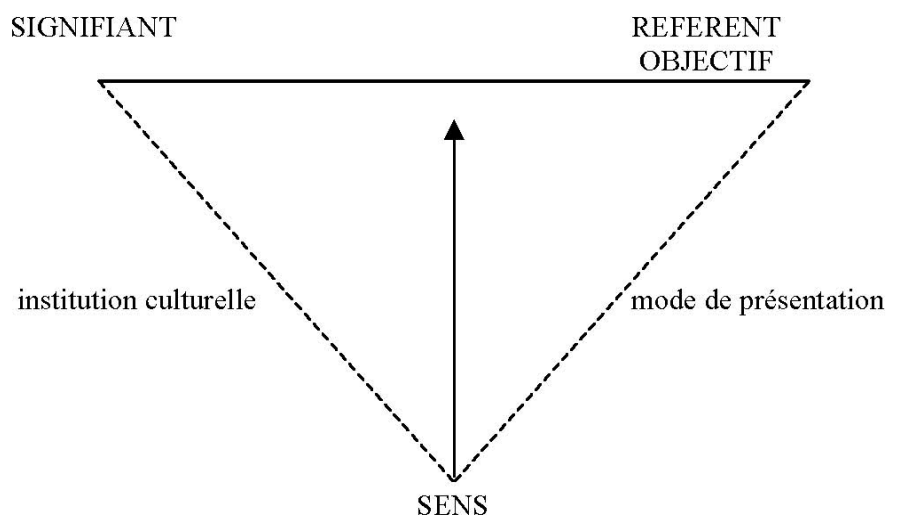

Figure 6

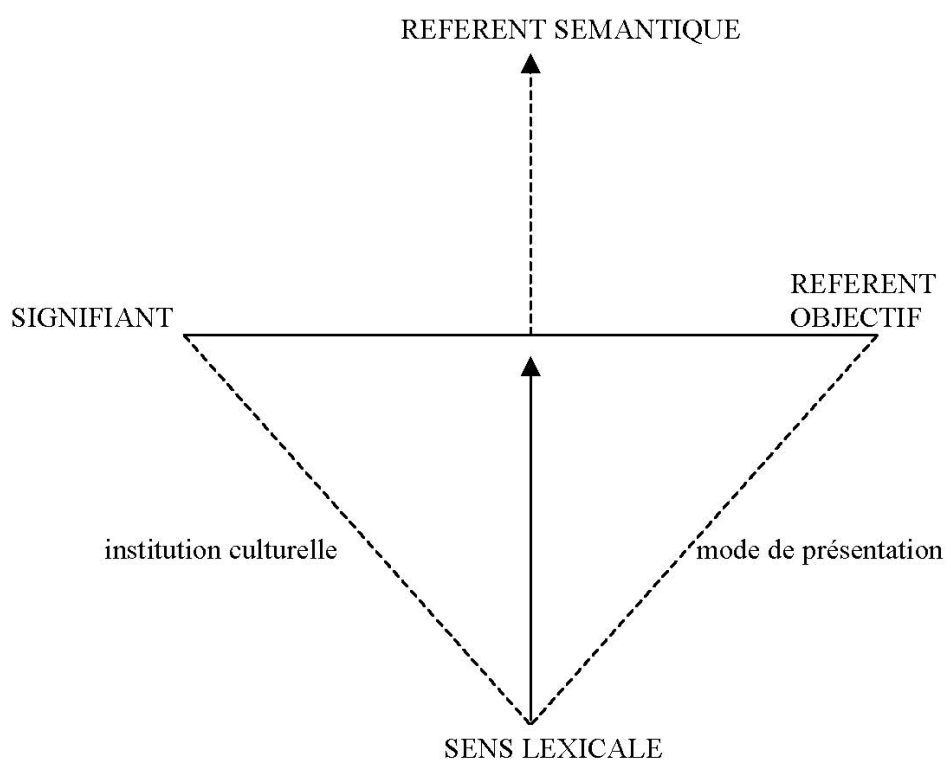

Figure 7

L'avant-dernier schéma (Figure 8), celui de Fromilhague et Sancier (1991), moins radical que les autres, révèle combien sont divergentes, par rapport à la conception saussurienne, les tentatives sémiotiques subséquentes. Pourtant, ces essais se révèlent prescients, tel que l'on le constate dans le deuxième schéma de Borella (Figure 7) où la pente entre le signifiant et le sens lexical a affaire à l'institution culturelle, nécessairement liée en terminologie à une société ou à un groupe socioprofessionnel qui la pratique. Pour atteindre ce qui est dénommé référent objectif au même niveau que le signifiant dans le schéma, les pratiques de l'institution coïncident avec le mode de présentation. C'est ce qui nous conduit dans la prochaine section aux réflexions de Diki-Kidiri (1999; 2000), parmi d'autres, qui explorent les faits culturels en terminologie. À titre d'exemple, dans un article paru dans Meta, Diki-Kidiri (1999: 573-581) se concentre sur la dynamique sens/signifiant à l'instar de Borella qui évoque dans son deuxième schéma plus haut la composante culturelle du rapport signifiant/sens. Pour paraphraser Diki-Kidiri dans l'article en question, bien que dans l'esprit humain les notions de «concept» et de «signifié » évoquent toutes les deux des produits culturels identiques, le concept paraît plus stable, tandis que le signifié, lui, semble plus intimement lié aux perceptions particulières à chaque culture.

\subsubsection{Tamba-Mecz (1991)}

Pour clore cette rétrospective sur la géométrie sémantique, nous reproduisons en dernier lieu le schéma (Figure 9) de Tamba-Mecz (1991) dont le motif sémiotique cherche à mieux aper- 
cevoir le rôle du pivot formel du mot lexical. En décrochant le signifié du concept, et le référent de la chose, pour présenter le signifié et le référent au moyen d'une seule et même forme (morphème lexical), le mot permet d'instaurer « un ordre de signification linguistique propre ». Et selon Gordon (1992: 345), ce modèle de Tamba-Mecz « renferme et dépasse le schéma d'Ogden et Richards », tout en voulant « corriger les abus du passé et du présent ».

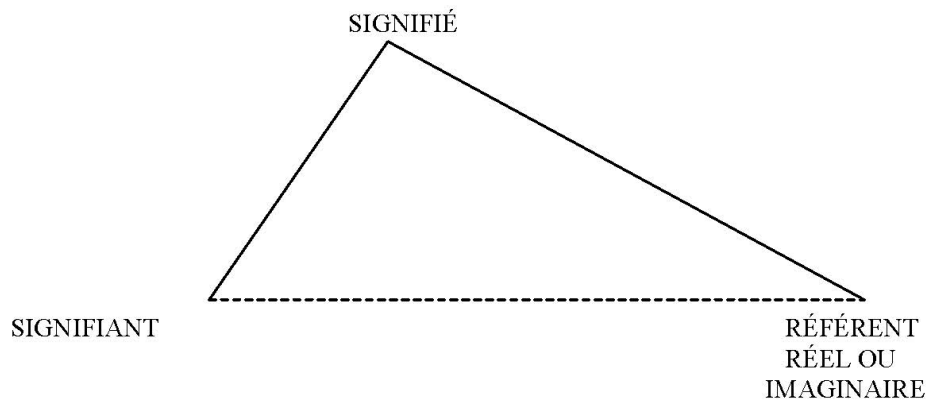

Figure 8

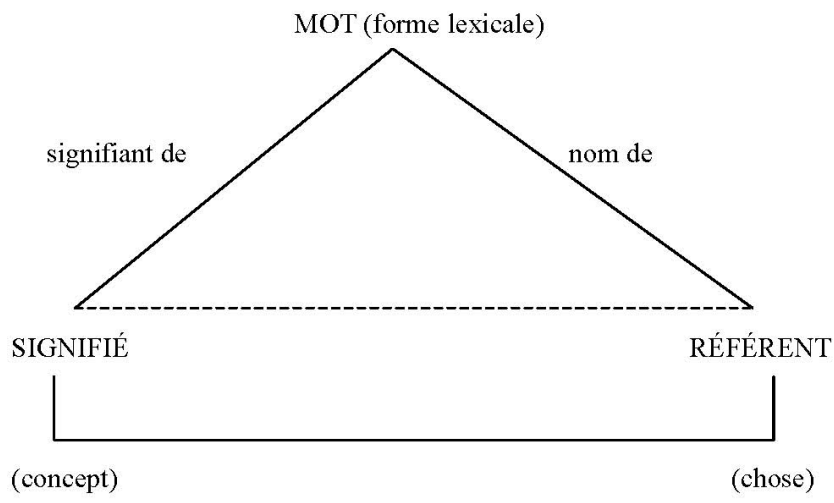

Figure 9

\section{$2.4 \quad$ Terminologue culturelle (Diki-Kidiri)}

Les tendances théoriques précédentes s'enracinent dans la volonté d'étendre non seulement la définition du terme comme unité primordiale de la terminologie, mais aussi de chercher par surcroît une description plus souple, adaptable et complémentaire dans les instances terminologiques diverses. Ainsi la terminologie culturelle évolue-t-elle vers la culture d'une communauté humaine, le véritable lieu de rencontre de diverses expériences humaines provoquant la production de savoirs et de savoir-faire de toutes variétés (Diki-Kadiri 2000). L'approche dite culturelle de Diki-Kidiri s'adapte particulièrement bien aux défis auxquels est confrontée la terminologie en langues africaines. Elle envisage une terminologie qui sera « une discipline autant de construction du savoir que de son appropriation à une culture particulière ». Il s'agit d'un domaine conscientisé aux fondements théoriques, aux méthodes, et aux dimensions socioculturelles, historiques, phénoménologiques et psychologiques, en même temps qu'il développe davantage ses visées linguistiques et techniques. Diki-Kidiri (2000: 30) souligne donc que l'Afrique, terrain fertile à part entière, offre une grande richesse d'observation de l'appropriation des réalités nouvelles, et qu'elle abrite autant de « traditions ancestrales extrêmement riches et vivantes ». Elle pourrait en conséquence se moderniser en empruntant à l'Occident des modèles qui lui conviennent le mieux.

Les nouvelles réalités perçant l'univers des diverses communautés culturelles africaines transforment celles-ci en partie intégrante d'un univers renouvelé et enrichi. De fait, la diversité des cultures est visée par ce nouveau jour sous lequel s'entreprend une plus profonde connaissan- 
ce de la culture mondiale. Le cas des langues africaines s'inscrit catégoriquement dans cette problématique, et force est d'atteindre le cadre théorique où pourra se réaliser une appréhension permettant de concevoir le terme de façon triangulaire : signifiant, signifié (sémantique lexicale), et concepts culturels englobant éventuellement les classes d'objet.

\section{$3 \quad$ La sémasiologie et la lexicologie}

D'après Cabré (1998: 30f.), les lexicographes partent de la dénomination, qui est une entrée de dictionnaire, pour la définir en se déplaçant du terme vers la notion. Ce faisant, la lexicologie et la lexicographie privilégient la sémasiologie parce que: (i) pour les lexicographes, l'exercice comprend en premier lieu le répertoire des unités, puis l'attribution de sens et d'équivalents; (ii) pour les usagers du lexique général, tracer le lien entre la forme et le sens implique souvent la recherche du sens ou de l'équivalent d'une unité en partant de sa forme graphique ou sonore immédiatement à leur portée.

\subsection{La sémiasiologie et la définition lexicographique}

Suite à sa filiation et à son historique, la sémasiologie est grosso modo la science linguistique de recherche de définitions attribuées à une unité linguistique. Dans ce sens, en prenant le signifiant comme l'entrée de dictionnaire, la définition de cette unité de dictionnaire devient l'explicitation du sémème, soit l'unité constituée du signifiant et du signifié. Ainsi paraît-elle évidente la relation entre la sémasiologie et la définition. Depuis Platon et Aristote, nous le révèle Gavoille (1999: 82), les « rhéteurs et les philosophes s'accordent sur la fonction générale de la définition: lever l'ambiguitté, éviter le quiproquo ». En citant Aristote au sujet de l'exigence de la concision et de l'exhaustivité dans la définition, Gavoille (1999: 82) ajoute que «la définition embrasse d'une façon concise et complète les qualités spécifiques d'une chose ».

La définition est un outil indispensable en terminographie, aussi bien qu'en lexicographie, parce qu'elle explicite la signification, les qualités et les propriétés de chaque unité avec un degré reconnaissable de clarté et de concision. En lexicographie, la sémasiologie est la recherche de la définition ou de l'explicitation d'un mot. La définition d'un mot est une explication des qualités, des caractères et des sens du mot en visant la notion ou le concept que représente ce mot. Le sens accordé à un mot est gouverné par la convention, l'usage et l'intelligence commune d'une langue donnée.

Gavoille (1999: 84) distingue entre la définition descriptive et la définition prescriptive ou constructive. Sur le plan sémasiologique, la définition descriptive consiste à définir un mot en rappelant son usage tandis que, si elle est constructive, la définition vise à éclaircir le concept de la chose représentée par le mot. Souvent, les lexicographes accordent à la fois la définition descriptive et la définition prescriptive à l'unité de dénomination. Dans le premier cas, l'accent porte sur la prononciation, l'usage ponctuel et d'autres caractéristiques du signifiant, tandis que dans le cas prescriptif, le mot est relié à un signifié qui l'explique. D'une perspective sémasiologique, c'est la description prescriptive qui révèle le rapport entre le mot et son sens étant donné que les variétés de significations du mot sont explicitées. On recherche l'explication d'un mot, c'est-à-dire, son signifié, à partir du mot lui-même, ce qui situe cette démarche dans le domaine de la sémasiologie.

Ainsi la définition vise-t-elle à dévoiler une idée cachée représentée par un mot en isolant le rapport entre le mot et ses significations. À ce sujet, selon Gavoille (1999: 86), « définir c'est mettre à découvert ce qui est enveloppé, caché [...], sous le mot». Dans cette optique, Rey (1965: 71, cité dans Lehmann 1990: 209) nous rappelle que «le programme de la définition se réduit, pour la pratique lexicographique, à faire correspondre à une unité lexicale supposée inconnue ou mal connue une pluralité d'unités [...] qui sont supposées (1) capables de ren- 
voyer au même signifié et (2) capables de déterminer chez le lecteur ou l'auditeur l'élaboration conceptuelle de ce signifié ». En faisant allusion à Robinson (1950, cf. Gavoille 1999: 87), nous distinguons les types de définition suivants :

\subsection{La définition terminologique}

Considérer la définition du point de vue précédent, c'est l'accepter sous un jour sémasiologique, ce qui fait croire que la définition appartient à la lexicologie et à la lexicographie. Cette axiomatisation se déduit pareillement du questionnement de De Bessé (1990: 252) qui s'interroge sur l'existence d'une définition terminologique en se posant la question suivante: «En quoi diffère-t-elle de la définition lexicographique et de la définition encyclopédique? ». De Bessé conclut qu'il existe vraiment une définition terminologique, ce qu'il croit avoir justifié en montrant que «la banque de terminologie du Canada, TERMIUM, contient près de 900 000 fiches bilingues comprenant une définition ». Par ailleurs, on admet généralement que les définitions terminologiques se distinguent des définitions lexicographiques, raison pour laquelle certains linguistes-terminologues parlent de la lexicographie spécialisée. De façon générale, les définitions terminologiques s'appliquent strictement aux termes; autrement dit, les unités lexicales ayant acquis des définitions technoscientifiques appartenant aux domaines spécialisés et par opposition au lexique général.

La définition lexicographique et la définition terminologique se distinguent d'abord selon les objets qu'elles visent, pour ne rien dire des méthodologies qu'elles fréquentent. L'objet primordial que traite la lexicographie est le mot et son sens, tandis que la définition terminologique ou terminographique a trait principalement au terme et au concept. Lorsque défini d'une approche sémasiologique, le rapport mot-sens fait preuve d'une équivocation sémantique face à la polysémie et à d'autres incidences connotatives. Par contre, le rapport terme-concept fait preuve de l'univocité du point de vue sémasiologique, mais de la biunivocité du point de vue onomasiologique. Toutefois, le fait que la terminologie part théoriquement du concept vers la forme du terme n'exclut point toute possibilité d'une définition du terme ou du concept.

Un terme désigne un concept qu'il distingue de tous les autres concepts du même système conceptuel. Selon De Bessé (1990: 253), «le lien entre la définition et le terme est un des fondements de la terminologie et de la terminographie » parce qu'« on ne peut utiliser de langage scientifique sans définitions ». La grande différence entre la définition lexicographique et la définition terminologique ou terminographique est basée sur leur point d'entrée. La définition lexicographique se fonde sur le mot pour en expliciter les acceptions, ce qui lui attribue sa qualité sémasiologique. Par contre, la définition terminologique/terminographique privilégie le concept en rapport avec sa représentation linguistique.

Une terminologie sémasiologique appréhendée par le biais de la définition, imputable normalement à la lexicologie, n'est ni farfelue ni lointaine. Du point de vue onomasiologique, la définition n'est pas le concept lui-même, mais plutôt une explication du concept, le concept considéré comme étant un élément antérieur au terme. Une approche strictement onomasiologique serait insuffisante, étant donné que le concept est déjà délimité soit par le domaine, soit par les instances institutionnelles. Il est possible d'argumenter, du point de vue onomasiologique, que le terme est une définition du concept, mais puisque ce ne serait pas toujours le cas, le coté pratique de la discipline révèle qu'un terme représente un concept qui est explicité par une définition. Donc, accepter l'existence d'une définition terminologique, c'est admettre qu'il existe une terminologie à caractère sémasiologique.

\subsection{La définition terminologique et le concept}

Du point de vue sémasiologique, les lexicographes conçoivent le mot définir comme une explication du sens du mot. En revanche, les terminologues, comme les terminographes, conçoi- 
vent la définition comme ce qui peut « décrire, délimiter et distinguer des concepts » (De Bessé 1990: 254). Les termes d'un domaine donné constituent un système définitionnel, classificatoire, hiérarchisant et structurant les concepts du domaine, car tous les termes appartenant à un domaine constituent son système terminologique auquel accéder par son ontologie qui en définit le système des concepts bien organisés et bien structurés. La définition terminologique ressemble donc à la définition encyclopédique, car toutes les deux partent de l'extralinguistique, mais la définition terminologique s'arrête dès que sont élucidés les traits qui distinguent un concept de tous les autres du même système conceptuel.

\subsection{Le triangle terminologique}

De Bessé (1990: 255) postule donc que la relation biunivoque terme-concept ne représente pas vraiment l'image du rapport onomasiologique entre le concept et le terme qui dénomme celui-là. Tout comme Ogden et Richards qui ont ajouté un troisième aspect à la double facette saussurienne du signe linguistique (voire signifiant, signifié et référent), De Bessé ajoute un troisième élément à la représentation du concept (soit concept, définition et terme). Il les conçoit comme trois entités distinctes constituant la même chose. Du point de vue onomasiologique, les terminologues cherchent à accorder une dénomination à un concept, mais la dénomination n'est pas à substituer à la définition. La définition est l'explication qui différencie les différents concepts à l'intérieur d'un système conceptuel. Par exemple, le terme aérosol acide désigne un concept qui se définit comme : "dans un milieu gazeux, suspension sous forme solide ou liquide de particules acides finement divisés et présentant une vitesse de chute négligeable»(De Bessé 1990: 255). Cette représentation triangulaire implique que le concept est désigné par un terme, explicité par une définition, mais que tous les trois (concept, terme et définition) relèvent d'un domaine donné. Il serait donc futile de se demander en quoi la définition terminologique pourrait se distinguer d'une définition lexicographique banale, car de toute évidence le terme en question appartient incontestablement à un domaine restreint de savoir.

\section{$4 \quad$ Onomasiologie}

Pour l'onomasiologie, on distingue (Grzega 2003) l'orientation traditionnelle de celle récente et disciplinaire. Elle est perçue comme une sous-discipline de la linguistique cognitive car elle étudie le côté cognitif du rapport terme-concept. Par opposition à l'approche traditionnelle, l'onomasiologie cognitive dépasse la simple recherche de l'unité lexicale attribuée à un concept; elle permet aussi d'appréhender les processus de création de nouvelles unités linguistiques pour dénommer des concepts déjà existants ou ceux qui sont nouvellement explicités. Mais c'est Štekauer (2001: 2) qui établit un lien entre l'onomasiologie et la grammaire cognitive en affirmant que toutes les deux se ressemblent en ce sens qu'elles privilégient l'aspect sémantique et cognitif du lien entre le concept et l'unité lexicale par rapport à d'autres aspects de la linguistique tels que la phonologie et la phonétique. Du point de vue de la grammaire cognitive, les unités grammaticales sont des symboles ayant deux aspects complémentaires: l'aspect sémantique et l'aspect phonologique. Dans ce sens, le rapport onomasiologique entre le concept et le terme est un rapport de représentativité.

Toutefois, l'onomasiologie et la grammaire cognitive se distinguent dans leurs buts et leurs méthodologies. La grammaire cognitive s'intéresse au fonctionnement du système grammatical en général, en mettant l'accent sur le sens, tandis que l'onomasiologie ne s'y intéresse qu'en partie, dans la mesure où il s'agit du rapport entre le concept et l'unité de dénomination. À ce sujet, Štekauer (2001) remarque que la grammaire décrit le fonctionnement du système grammatical et celui des unités grammaticales, tandis que l'onomasiologie se préoccupe de la formation des unités lexicales saisies du point de vue du concept. Bien que l'onomasiologie ne soit pas elle-même le processus de création des nouvelles unités, elle étudie les différentes 
manières d'attribution de dénominations à des concepts, y compris la création de nouvelles unités lexicales. Donc, trois opérations sont impliquées dans le processus d'attribution de dénominations : le changement sémantique (la conversion), la créativité lexicale et l'emprunt.

\subsection{La conversion/Le changement sémantique}

Par néologisme sémantique (dit aussi conversion, quoiqu'elle se distingue ici du changement de catégorie morphosyntaxique pour un même item), il s'agit de créer un rapport entre un concept et un terme en attribuant à ce concept une unité ayant déjà une existence lexicale. Dès qu'un terme existant sera attribué à un concept, le rapport entre les deux entités pourra être étudié des deux perspectives onomasiologique et sémasiologique. Du point de vue sémasiologique, la conversion, autrement appelé le changement ou la modification sémantique, consiste en l'attribution d'un nouveau sens à un terme existant ayant déjà son propre sens. C'est le cas où une unité est empruntée pour modifier le sens selon le besoin dénominatif du domaine d'accueil. La conversion aboutit souvent à la polysémie en ce sens qu'un sens supplémentaire est automatiquement attribué à l'unité comprise dans cette opération.

\subsection{La créativité lexicale}

Prise dans le train de réflexion qui précède, la créativité lexicale relève de l'utilisation des ressources naturelles que possède une langue pour former de nouvelles unités lexicales permettant de dénommer de nouveaux concepts. Comme nous l'avons vu plus haut, l'onomasiologie, par opposition à la sémasiologie, se préoccupe du processus de créativité morphologique. Štekauer (2001: 4) postule donc que le terme onomasiologie est souvent employé comme antonyme du terme sémasiologie: le premier étudie les processus de création des nouvelles unités lexicales pour répondre aux besoins de dénomination résultant de nouvelles découvertes, tandis que le dernier se consacre à l'analyse des unités lexicales existantes. Pour cette raison, l'interaction entre l'onomasiologie et la sémasiologie en terminologie systématique devient pertinente.

\subsection{L'emprunt}

Par opposition à la mise ensemble des ressources que fournit naturellement une langue pour former de nouvelles unités de dénomination, l'emprunt est un processus qui pourra combler le vide créé par un besoin dénominatif. Au lieu d'une création ex nihilo, on emprunte des unités de dénomination à d'autres langues dans lesquelles le même concept aurait déjà été dénommé et stabilisé. D'une perspective sémasiologique, l'emprunt éventuel entraînera l'existence de plusieurs untés lexicales translinguistiques désignant le même concept au sein du même domaine.

\subsection{L'onomasiologie et la productivité lexicale}

Par sa nature dynamique, une langue devrait être productive, c'est-à-dire qu'elle devrait fournir des ressources linguistiques servant à la création de nouvelles unités selon le cas. Cette productivité lexicale est perceptiblement sujette aux règles grammaticales et aux modes de formation de mots. Cette capacité, que ce soit innée ou acquise, permet aux locuteurs d'enrichir le lexique. La productivité est donc un processus d'engraissement et de maintenance de la capacité cognitive et expressive des locuteurs.

\subsection{L'onomasiologie et la réalité extralinguistique}

Sur le plan onomasiologique, grâce à sa capacité cognitive la langue cerne la réalité extralinguistique à laquelle il faut attribuer une dénomination. On constate donc l'existence d'une interrelation entre le concept qui est un élément extralinguistique et l'unité lexicale qui est un 
élément du système linguistique. Le processus de dénomination d'un nouveau concept est précédé d'analyses conceptuelles de l'objet à dénommer, à la fin desquelles on parvient à sa propre catégorie conceptuelle : substance, action, processus, état, qualité, etc. L'onomasiologie est donc un mouvement qui part du système extralinguistique (conceptuel) vers le système linguistique (ou terminologique).

\section{$5 \quad$ L'onomasiologie et la sémasiologie en tandem}

L'onomasiologie et la sémasiologie s'opposent de par leurs différentes approches du rapport terme-concept. Toutefois, elles se complètent à bien des égards. On ne peut guère parler exhaustivement de l'une sans faire allusion à l'autre. Quant à la terminologie dont la modalité serait sémasiologique, il serait question de creuser les différents sens (polysémie) d'une même unité lexicale, mais sur le plan onomasiologique, on tracerait les voies lexicales par lesquelles un concept donné retrouverait la forme qui lui est attribuée (Figures 10 et 11).

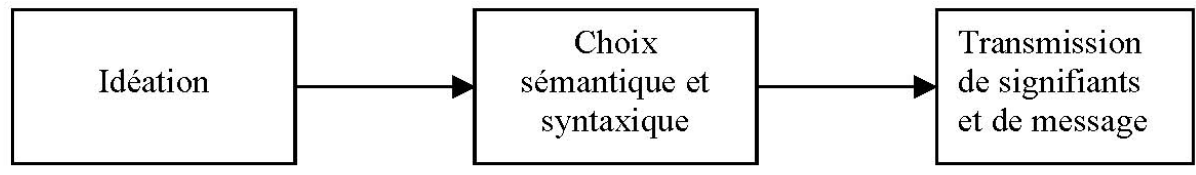

L'axe onomasiologique

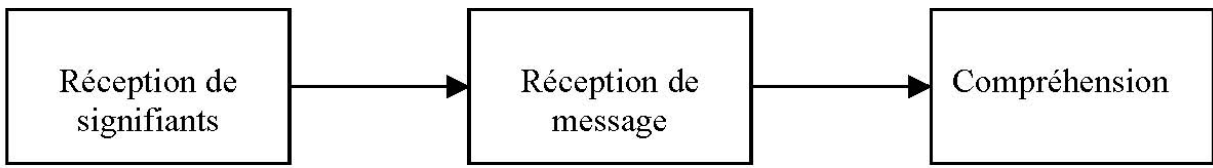

L'axe sémasiologique

Figure 10

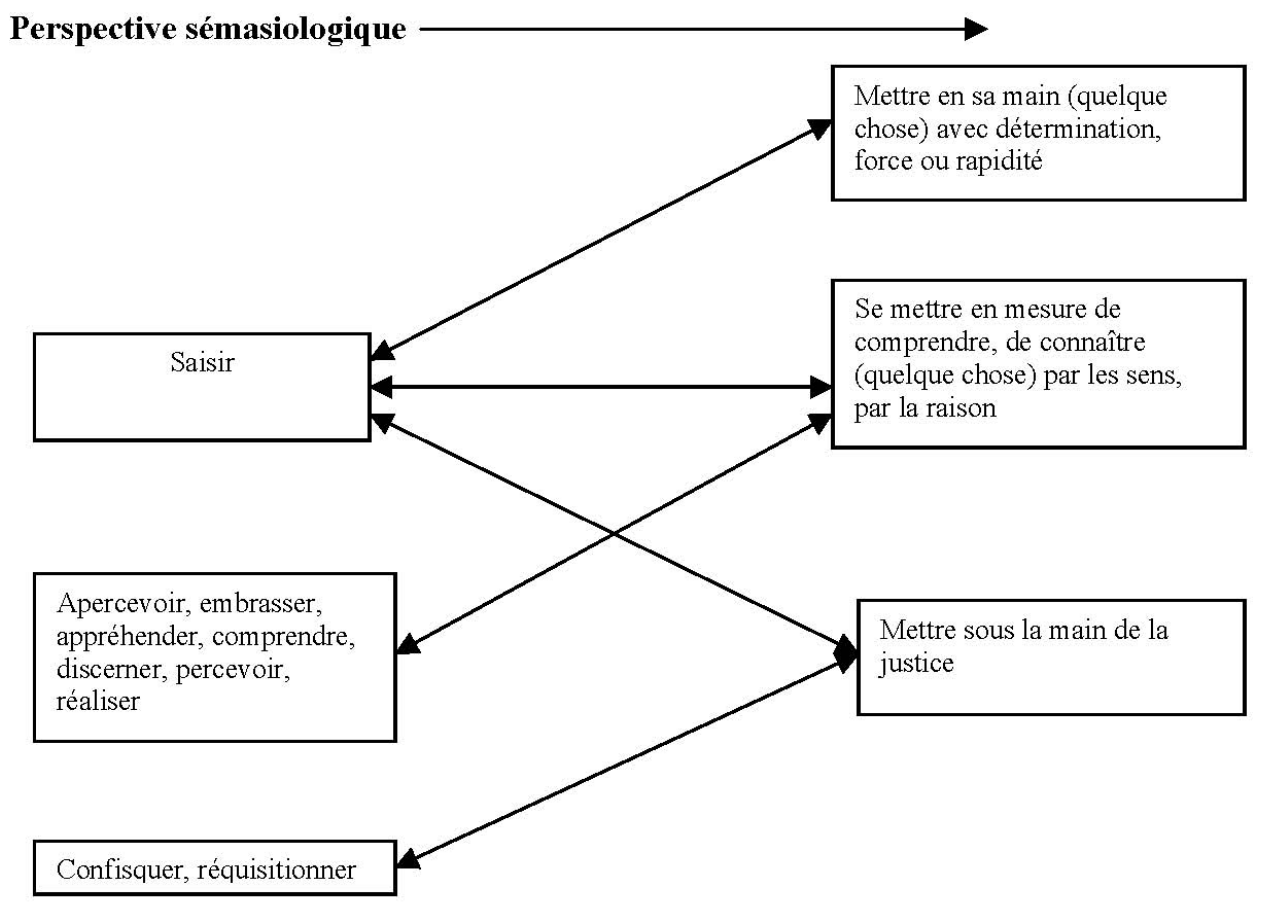

Perspective onomasiologique

Figure 11 


\section{$6 \quad$ Structure conceptuelle des unités complexes}

D'après L'Homme (1996: 25), l'unité terminologique syntagmatique complexe est susceptible de rendre plus explicite l'organisation conceptuelle des réalités qu'elle dénomme. Pour ce qui est de son rapport avec le concept, la plupart des termes complexes que nous avons analysés sont, par leur nature, transparents, étant donné que leur composition formelle explicite en quelque sorte la réalité qu'ils représentent. Les termes de l'informatique tels que traitement de données, circuit de lecture optique, imprimante à laser, lecture multiligne, levier de libération, levier de détente, caractère d'effacement de ligne, sont sémantiquement transparents du fait qu'il y a une motivation sémantique directe entre la forme et le concept qu'elle dénomme car, pour traitement de données par exemple, il s'agit d'un traitement qui s'applique aux données.

Ce qui précède nous conduit au rapport syntaxique entre les composants d'un terme syntagmatique, y compris des éléments joncteurs comme les prépositions que nous isolerons plus bas. Une des unités, la tête, garde un lien sémantique direct avec le concept désigné, tandis que le deuxième élément ne remplit que le rôle de modificateur ou de régisseur. Dans le cas de imprimante à laser, imprimante est la tête ou le régissant parce qu'il s'agit d'une imprimante. Laser (le régisseur), qui joue un rôle adjectival, distingue l'imprimante dont il est question de toutes autres imprimantes telles imprimante matricielle, imprimante à jet d'encre. La préposition $\grave{a}$, qui s'y intercale, relie les deux noms en indiquant un rapport, soit celui de $<$ fonctionner à l'aide de > : imprimante qui fonctionne à l'aide de laser; ou de <de type $>$ : imprimante de type laser.

Dans le cas des syntagmes nominaux lexicalisés de nature tandem (Aito 1999: 56), c'est-àdire les syntagmes qui comportent deux noms tels que carte modem, carte radio, carte son, carte réseau, carte vidéo, carte mère, il serait parfois difficile de préciser le terme régissant et le terme régisseur. La précision du régissant et du régisseur ne dépend pas de la classe grammaticale des composants, mais plutôt de facteurs sémantiques, du concept lui-même, ce qui permet de dégager la fonction grammaticale de chacun des composants. Dans le cas de carte réseau, par exemple, il s'agit dans sa définition d'une «carte d'extension mise en place dans un ordinateur pour le connecter sur un réseau local »(Voss 1999: 111). Ainsi, on dirait que carte est le régissant tandis que réseau est le régisseur (de même pour carte RAM, Carte TV, et carte VGA). En revanche, il n'en va pas de même dans le cas de carte modem parce qu'il ne s'agit plus simplement d'une carte, mais plutôt d'un «type de modem qui se présente sous la forme d'une carte d'extension à enficher à l'intérieur de l'ordinateur » (Voss 1999: 110). Cela ne nous empêche pas d'avouer que cette différenciation parait quelque peu douteuse, car il s'agit de cartes à fonction spécifique. Ces pseudo-composés fonctionneraient de façon inverse dans d'autres langues telles l'anglais et l'allemand où le déterminé précéderait le déterminant.

\subsection{Le traitement sémasiologique des prépositions}

Il ne s'agit pas ici d'unités simples (constituées d'un seul nom) de type ordinateur, disquette, ni de termes tandems, mais plutôt d'unités complexes, soit des syntagmes lexicalisés (comportant au moins deux noms rapprochés par l'entremise d'une préposition) de type système de gestion de base de données, traitement de texte, traitement des données, traitement de texte automatisé, traitement de texte banalisé, traitement de texte intégré. On y constate la place qu'occupe la préposition en tant que connecteur syntaxique, ce qui donne une formation de type $N+$ Prép $+N$, et $N+$ Prép $+N+A d j$. De nos items, on extrait deux catégories de préposition: les prépositions pluricorrélatives ( $a$, de et $e n=$ qui expriment plusieurs rapports) et les prépositions monocorrélatives (par, sans, pour = qui expriment souvent un seul rapport) (L'Homme 1996). 
Dans les syntagmes lexicalisés, les prépositions se prêtent plus à l'analyse sémasiologique (textuelle et lexicale) qu'à l'analyse onomasiologique. C'est parce que l'intérêt ne porte pas souvent sur le sémantisme individuel des composants d'un syntagme lexicalisé. Par exemple, dans le terme traitement de données, le terme est pris comme une entité qui représente un seul concept, et non l'ensemble des concepts attribuables aux trois unités lexicales.

\subsection{Les prépositions pluricorrélatives}

Nos interrogations actuelles, encore irrésolues, nous mènent à la catégorisation des prépositions dont la fréquence n'est pas négligeable. Pourrait-on fonder leur fonctionnement sur une simple approximation face à la grammaire du français? Nous espérons y trouver une issue quand nous aurons établi notre corpus. Pour le moment, nous les partageons et les décrivons ci-bas.

\section{A. La préposition « à » exprime les variétés de rapports suivantes:}

1. «Fonctionner à l'aide de » ou « de type »: La préposition à indique un rapport de type «fonctionner à l'aide de » ou « de type » dans les termes câble à fibres optique « un support moderne de transmission fonctionnant par effet optique » (Voss 1999: 95); câble à paires torsadées, "câble constitué d'une ou de plusieurs paires torsadées qui comportent chacune deux fils isolés, enroulés l'un sur l'autre, afin de réduire les interférences électromagnétiques » (GDT). D'autres exemples sont organe à accès série (angl. serial access device), et souris à connexion série.

2. «Comporter »: Ce type de rapport se voit dans les termes câble à paires, « câble contenant un certain nombre de paires torsadées qui ne sont pas associées pour former des quartes »(GDT); alternateur à fer tournant, code à sept positions, fichier à accès séquentiel, logique à seuil élevé, circuit à seuil, opération à un seul pas (angl. one-step operation).

3. «Destiné à »: On peut constater ce rapport dans les termes boîte à confettis, «boîte qui sert à recueillir les confettis qui se détachent des supports de données ou d'informations comme les cartes, les bandes perforées, les bordures de papier d'impression en continu, après que l'on y a perforé des trous »(GDT); boîte à outils, boîte aux lettres.

B. La préposition « de » exprime les différents rapports suivants:

1. «Agit sur»: Ce type de relation se voit dans les termes suivants : modification d'adresse, transformation d'application.

2. «Sert à »: Ce genre de rapport existe entre les composants joints par la préposition de: système de gestion de bases, système de fichiers (un système qui «autorise les processus à accéder aux données, en série parallèle » (Voss 1999), système de développement, carte de recouvrement, adaptateur de signal, seuil de commutation, seuil de luminosité (angl. light threshold).

3. «Composé de »: Sémantiquement, dans les termes suivants, le type de rapport exprimé par la préposition est celui qui révèle que le régissant est composé du régisseur chaîne de caractères, fil de plomb.

4. « Appartient à »: La préposition de peut aussi relier les deux éléments d'un terme complexe de façon à ce que le régissant appartienne au régisseur. C'est la sorte de rapport exprimé dans les termes de type : racine de lotus, tuyau de cheminée.

5. «Au sujet de »: On constate aussi des cas où la préposition indique des rapports sémantiques selon lesquels le sens du régissant s'applique à celui du régisseur, comme dans les termes suivants : avis d'évaluation, registre des absences. 


\section{La préposition « en»:}

Trois rapports principaux sont possibles à déceler, selon la classe conceptuelle des composants du terme. Lorsque le régissant dénote un objet et le modificateur, une substance, le rapport exprimé est celui de « composé de », par exemple, signal en double courant (angl. polar signal), télégraphie en double tonalité (angl. two-tone telegraph), registre en double mot (angl. double word register), mémoire image en double zone tampon (angl. double-buffered pixel memory). Si le régissant est un objet et le modificateur un concept de représentation, le rapport est alors «à la forme de " (L'Homme 1996: 88), par exemple, compteur en cascade (angl. cascadable counter), fenêtres en cascade (angl. cascading windows), montage d'amplificateur en cascade (angl. cascade amplifier). La préposition en exprime aussi le rapport « qui se trouve à », c'est-à-dire que le régissant se trouve au sein du régisseur, par exemple, dans les termes : caractère en bas de casse, caractère en haut de casse, lettre en bas de casse, lettre en haut de casse.

\subsection{Les prépositions monocorrélatives}

A. La préposition «par» exprime le seul rapport suivant:

«Fonctionner à l'aide de »: La préposition peut aussi relier les composants d'un terme complexe de façon à ce que, sur le plan conceptuel, le régissant se réalise au moyen du modificateur. Du point de vue sémantique ou conceptuel, dans le syntagme animation par ordinateur, il est évident qu'il s'agit d'une animation qui se fait à l'aide d'un ordinateur. D'autres exemples sont les termes où existe ce rapport sont: animation par image clé, transport par avion, perte par propagation, perte par absorption, perte par connexion et calculateur géré par programme.

B. La préposition « sans » exprime le seul rapport suivant:

« Ne comporte pas »: Les termes gant sans latex, téléphone sans fil, etc., exemplifient ce type de rapport sémantique. Il s'agit, en guise d'exemplification, d'un gant ne comportant aucun latex.

C. La préposition « pour » exprime le seul rapport suivant:

« Destiné à » : Pour exemplifier ce type de rapport, on notera que dans le terme marmite pour sauce, il s'agit d'une marmite qui est spécialement fabriquée pour servir la sauce. Voici d'autres exemple: histoire pour enfants, reliure pour imprimés (angl. printout binder), reliure pour imprimés détachés (angl. burst printout binder).

\section{$7 \quad$ Analyse conceptuelle des termes complexes}

L'analyse conceptuelle est une solution proposée pour combler les lacunes provoquées par la méthode classique de traitement des termes complexes en traduction. Il s'agit de tracer (i) la classe conceptuelle à laquelle appartiennent le régissant et le régisseur, et (ii) la corrélation entre les deux entités. Par exemple, le terme imprimante à laser peut suivre les différents niveaux d'analyse conceptuelle suivants :

(a) imprimante $=$ entité matérielle inanimée

(b) laser $=$ entité matérielle inanimée

Le régissant imprimante peut prendre n'importe quel autre régisseur, par exemple, imprimante à jet d'encre ou imprimante matricielle, etc. Le rapport exprimé, indiqué par la préposition « à », est celui de « fonctionner à l'aide de » (imprimante fonctionnant à l'aide d'un dispositif laser). 
Pour citer L'Homme (1996: 29), qui soulève des questions des prépositions et leur structure conceptuelle dans les termes complexes, « les mécanismes théoriques existant pour représenter les concepts et leur organisation, d'une part, et pour rendre compte de leurs rapports avec les mots, d'autre part, sont encore imparfaits ». Cela explique partiellement pourquoi dans le même domaine un concept peut finir par apparaître dans deux classes conceptuelles contiguës selon l'analyste.

\subsection{L'organisation conceptuelle}

Nous nous sommes jusqu'ici infléchis aux rapports conceptuels que facilitent les joncteurs à l'intérieur des termes complexes, rapports qui, dans notre estimation, sont facilement repérables dès l'analyse des formants et de leur rapport avec les concepts auxquels ils sont reliés. Nous nous servirons à ce stade de deux schémas (Figures 12 et 13) et d'autres exemples pour bien asseoir la proposition d'une terminologie à caractère sémasiologique. À cette fin, nous avons privilégié le terme siglé, $C D-R O M$, et ses dérivés.

Comme nous l'avons indiqué à maints endroits, grâce à la relation hyponymique ou hyperonymique qu'entretiennent les concepts au sein d'un domaine, tel que celui de l'informatique, il est possible d'organiser de façon hiérarchique les différentes relations sémantiques. Cette organisation pourrait être verticale (lorsque les concepts sont traités par rapport à un concept générique dont ils sont les différentes parties ou les différentes espèces), ou horizontale (lorsqu'on traite le rapport entre ces différentes parties ou ces différentes espèces d'un même concept générique). Du fait que CD-ROM à la demande, $C D-R O M$ à la norme $M P C, C D$ ROM de démarrage, CD-ROM d'accompagnement, CD-ROM de formation, sont des espèces du concept générique $C D-R O M$, la relation entre ces concepts par rapport au concept générique est représentée de façon verticale, soit du générique vers ses parties ou ses espèces. 


\section{Organisation verticale des concepts}

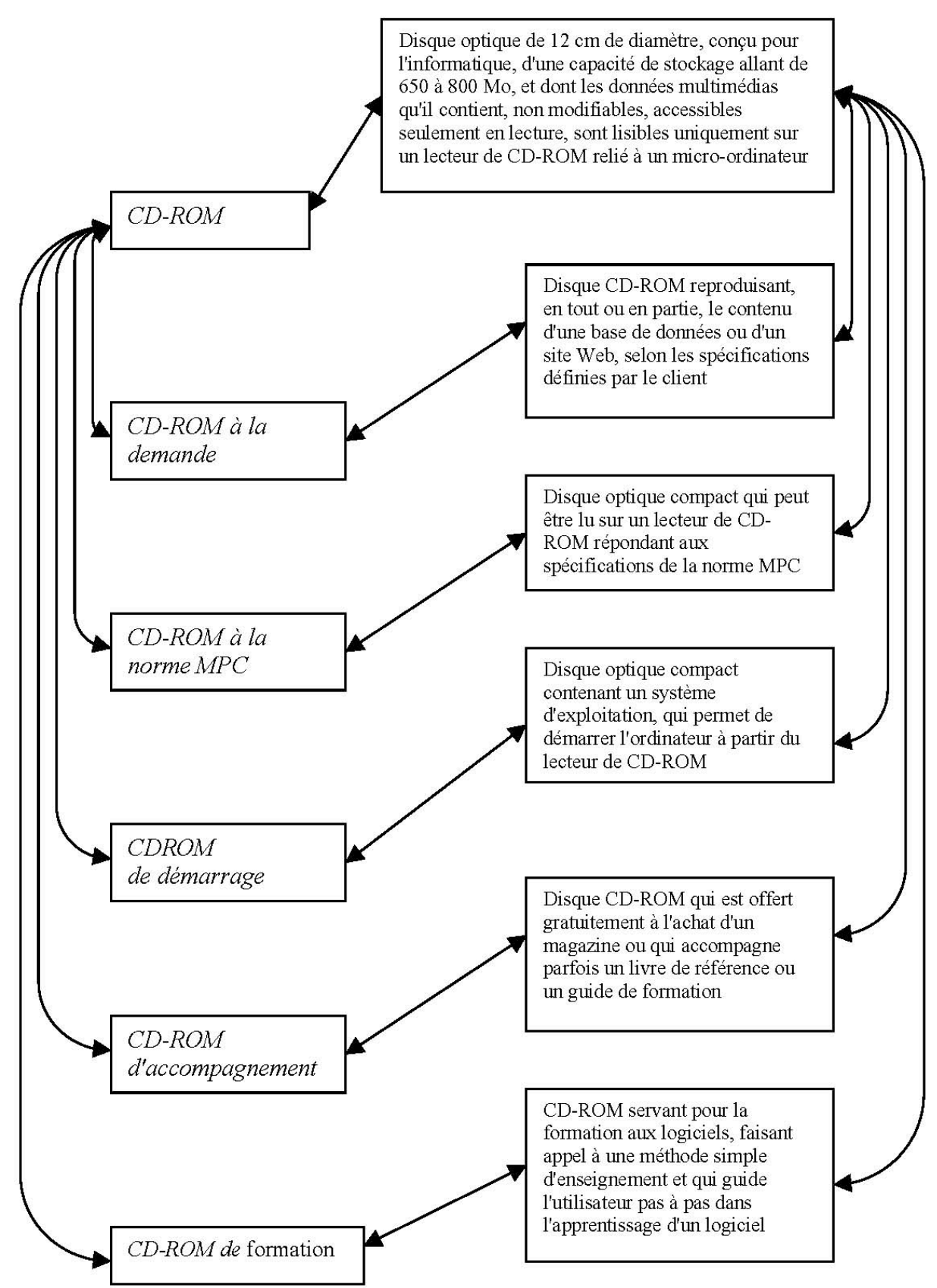

Figure 12

Comme on peut l'observer dans la Figure 12, les flèches onomasiologiques qui descendent vers les termes relient chaque concept à un terme sans sensiblement permettre de constater le rapport morphologique entre le terme et un autre. Il faut donc une approche basée sur le côté lexical pour révéler la relation morphologique entre les termes $C D-R O M$ à la demande, $C D$ ROM à la norme MPC, CDROM de démarrage, CD-ROM d'accompagnement, et CD-ROM de formation, qui sont formés à partir du générique $C D-R O M$. La terminologie sémasiologique s'avère donc pertinente, car une meilleure compréhension des aspects lexicaux permettra sans doute une meilleure saisie de la dynamique conceptuelle.

L'organisation horizontale (Figure 13) représente la relation entre les concepts qui sont des espèces ou des parties d'un même concept. Il s'agit d'une relation d'appartenance, de coordination ou de subordination. Les flèches bidirectionnelles indiquent que le rapport entre les 
concepts et les termes peuvent être considérés des deux perspectives onomasiologique et sémasiologique.

Organisation horizontale des concepts

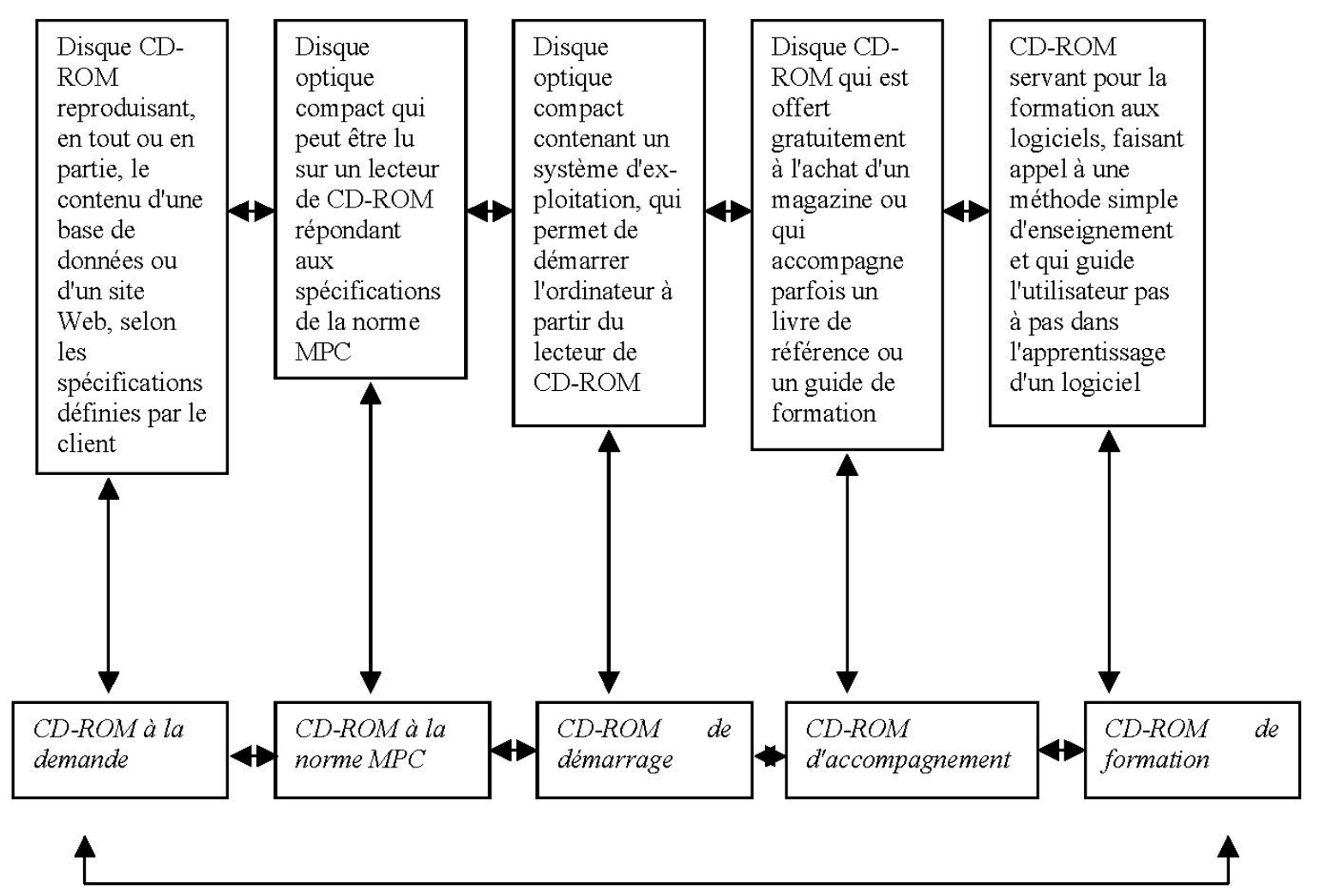

Figure 13

\section{Conclusion}

L'ensemble des termes s'articulant autour d'un concept générique qui se distinguent par certaines nuances sémantiques constitue un champ sémantique. Le champ sémantique lui-même est constitué d'un champ lexical et d'un champ conceptuel (Schwischay 2001). Deux opérations distinctes sont comprises dans l'élaboration d'un champ sémantique telle que nous l'avons fait avec les Figures 12 et 13. Il s'agit donc de la constitution du champ sémantique, suivie de sa description. La constitution d'un champ sémantique se fait normalement selon une démarche onomasiologique. Pour ce faire, constituer un champ sémantique, l'opération consiste à délimiter préalablement le champ conceptuel, puis à sélectionner les termes (le champ lexical) ayant trait à ces concepts délimités. Il faut premièrement délimiter le concept générique grâce auquel l'on pourrait récupérer tous les autres concepts spécifiques qui lui sont apparentés.

Du point de vue lexical, si on se limite à l'analyse onomasiologique du rapport entre les concepts et les termes d'un domaine, on risquera de négliger les différentes fonctions morphologiques, sémantiques et grammaticales que remplissent les différents composants de ces termes. Ceci parce que l'onomasiologie est un procédé qui traite d'un concept donné pour un terme donné. Il faudra donc une approche mixte (onomasiologique et sémasiologique) pour mieux cerner le processus de la construction de sens en même temps que celui de la construction morphologique (Aito 2004).

Si nous avons repris plus haut la question des prépositions dans les termes syntagmatiques, c'est que la terminologie foisonne d'unités terminologiques lexicalisées complexes. Nous avons donc voulu nous en servir pour démontrer le bien-fondé éventuel d'une terminologie sémasiologique. Les deux faces du signe linguistique, à savoir le signifiant et le signifié, inté- 
ressent la terminologie. L'approche qui part du signifiant vers le signifié implique un processus vers le concept à partir de la forme que l'on a sous le nez. Cet axe est donc sémasiologique. Il est relatif à la lexicologie et à la lexicographie dont l'exercice comprend d'abord le répertoire des signifiants, puis l'attribution des signifiés. Le deuxième axe, soit celui qui repose sur l'onomasiologie, procède du concept vers la forme phonique et graphique. Cette approche relève de la terminologie et de la terminographie où il est question de systématiser les concepts qui constituent éventuellement son ontologie.

En réalité, les pratiques terminologiques sont également définitionnelles, mais elles pourraient profiter davantage des deux démarches onomasiologique et sémasiologique. Autrement dit, concrètement, une approche hybride qui exploite les zones limitrophes des deux approches.

\section{Bibliographie}

Aito, Emmanuel (2006): "L'unité terminologique et les langues en voie de développement: réflexions épistémologiques et ontologiques". Cahiers du rifal 25: 6-16.

Aito, Emmanuel (2004): "Morphologie dérivationnelle et construction de sens". Linguistik Online 19/2: 3-13.

Aito, Emmanuel (1999): "La conversion et l'interface infinitif/impératif/nom dans la terminologie du micro-ordinateur". Terminologies nouvelles 20: 54-59

Auger, Pierre (1988): "La terminologie au Québec et dans le monde, de la naissance à la maturité". In: Gaumond, Jean-Claude (ed.): L'ère nouvelle de la terminologie. Actes du $6^{e} \mathrm{col}$ loque OLF-STQ de terminologie. Montréal, OLF: 27-59.

Auger, Pierre/L'Homme, Marie-Claude (1994): "La terminologie selon une approche textuelle : une représenatation plus adéquate du lexique dans les langues de spécialité". ALFA 7/8: $17-21$.

Boulanger, Jean-Claude/L'Homme, Marie-Claude (1991): "Les technolectes dans la pratique dictionnairique générale : quelques fragments d'une culture". Meta 36/1: 23-40.

Borella, Jean (1989): Le mystère du signe: histoire et théorie du symbole. Paris: Éditions Maisonnette \& Larose.

Cabré, Teresa Maria (2000): "Terminologie et linguistique : la théorie des portes". Terminologies nouvelles 21: 10-15.

Cabré, Teresa Maria (1998): La Terminologie : théorie, méthode et applications. Ottawa: Presses de l'Université d'Ottawa/Paris: Masson et Armand Colin.

De Bessé, Bruno (1990): "La définition terminologique". In: Chaurand, Jacques/Mazière, Francine (eds.): La définition. Paris, Librairie Larousse: 252-261.

Diki-Kidiri, Marcel (1999): "Le signifié et le concept dans la denomination". Meta XLIV, 4: 573-581.

Diki-Kidiri, Marcel (2000): "Une approche culturelle de la terminologie". Terminologies nouvelles 21: 27-31.

Felber, Helmut (1987): Manuel de terminologie. Paris: UNESCO.

Felber, Helmut (1984): Terminology Manual. Paris: UNESCO.

Fromilhague, Catherine/Sancier, Anne (1991): Introduction à l'analyse stylistique. Paris: Bordas.

Gavoille, Elisabeth (1999): "Sens et définition chez Cicéron". In: Baratin, Marc/Moussy, Claude (eds.): Lingua Latina, Colloque du Centre Alfred Ernout. Paris, Presses de l'Université de Paris-Sorbonne: 81-95.

Gordon, W. Terrence (1994): "68 ans de géométrie sémantique". In: Phlipponneau, Catherine (ed.): Sociolinguistique et aménagement des langues. Moncton, Centre de recherche en lingustique appliquée, Université de Moncton: 333-347.

Garza-Cuaron, Beatriz (1991): Connotation and Meaning. Berlin: Mouton de Gruyter. 
Gordon, W. Terrence (1992): "68 ans de géométrie sémantique". Papers from the Annual Meetings of the Atlantic Provinces Linguistic Association (PAMAPLA) 16: 333-347.

Grzega, Joachim (2003): "Borrowing as a Word-Finding Process in Cognitive Historical Onomasiology". Onomasiology Online 4: 22-42.

Heger, Claus (1969): "L'analyse sémantique du signe linguistique". Langue française 1: 4466.

Hockett, Charles (1977): "Review of Thomas A. Sebeok (ed.): Current Trends in Linguistics, vol 12 (The Hague: Mouton, 1974)". Current Anthropology 18: 78-82.

Kageura, Kyo (1995): "Terminological Semantics: An Examination of Concept and Meaning in the Study of Terms". International Forum on Information and Documentation 20/4: 2531 .

Kocourek, Rostislav (1994): "Les textes spécialisés et la terminologie en tant qu'objet de l'analyse linguistique". ALFA 7/8: 9-14.

Lehmann, Alise (1990): "De définition à définition - l'interprétation dans le dictionnaire par le jeu des renvois". In: Chaurand, Jacques/Mazière, Francine (eds.): La définition. Paris, Librairie Larousse: 208-224.

L'Homme, Marie-Claude (1996): "Sélection des prépositions dans les termes complexes nom (prép) nom à partir de leur structure conceptuelle". Cahiers de lexicologie 68/1: 25-43.

Ogden, Charles K./Richards, Ivor A. ([1923]/1989): The Meaning of Meaning. New York: Harcourt Brace Jovanovich.

Oesterle, John A. (1944): "The Problem of Meaning". The Thomist 6: 180-229.

Rey, Alain (1995): Essay on Terminology. Amsterdam/Philadelphia: John Benjamin.

Russo, John Paul (1989): I.A. Richards: His life and Work. Baltimore: John Hopkins University Press.

Schogt, Henry (1976): Sémantique synchronique: synonymie, homonymie, polysémie. Toronto: University of Toronto Press.

Schwischay, Bernd (2001): Syntaxe du français. http://www.home.uos.de/bschwisc/archives/archives.htm.

Štekauer, Pavol (2001): "Fundamental Principles of an Onomasiological Theory of English Word-Formation". Onomasiology Online 2: 1-42.

Tamba-Mecz, Irène (1991): La sémantique. Paris: Presses Universitaires de France.

Temmerman, Rita (2000): Towards new ways of terminology description: The sociocognitive approach. Amsterdam/Philadelphia: John Benjamins.

Temmerman, Rita (2000a): "Une théorie réaliste de la terminologie: le sociocognitivisme". Terminologies nouvelles 21: 58-64. 